\title{
La conciliación entre las exigencias del ámbito productivo y las condiciones sociofamiliares: estudio de caso de una empresa
}

\author{
Pedro López \\ Carlos Lozares
}

Universitat Autònoma de Barcelona. Departament de Sociologia

Grup d'Estudis Sociològics sobre la Vida Quotidiana i el Treball

08193 Bellaterra (Barcelona). Spain

Pedro.Lopez.Roldan@uab.cat

Carlos.Lozares.Colina@uab.cat

\section{Resumen}

Este artículo examina, en primer lugar, el grado de asociación o independencia que guardan entre sí el ámbito socioproductivo y las condiciones sociofamiliares y, en segundo lugar, la incidencia que la conjugación de dichos ámbitos tiene sobre las prácticas y responsabilidades del hogar, así como sobre la dificultad para llevarlas a cabo. Los resultados son reveladores de las tensiones y contradicciones que provienen de conjugar, en la vida de los trabajadores y las trabajadoras, individualmente y como pareja, las exigencias que provienen de dichos ámbitos y, por tanto, de conciliar sus estrategias, proyectos, actividades y la posibilidad de acuerdos.

Palabras clave: conciliación, trabajo productivo, temporalidades, trabajo reproductivo.

Abstract. The reconciliation of requirements in the productive sphere and socio-family conditions: a firm case study

This paper examines, first of all, the grade of association or independence there are between the socio-productive and the socio-family conditions and, secondly, the effect of the combination of such ambits upon the activities and responsibilities of the family life, and also over the difficulties to accomplish them. The conclusions show the tensions and contradictions that arise when the workers (individually and with their couples) need to combine the family life and the requirements of their jobs. Therefore, the workers need to conciliate their strategies, projects, activities and possibilities of agreement

Key words: conciliation, productive work, temporalities, housework.

\section{Sumario}

1. Cambios, planteamientos y supuestos

2. Metodología

3. Análisis
4. Interpretación y conclusiones

Bibliografía 
Este artículo retoma algunos contenidos parciales de una investigación más general llevada a cabo en una empresa ${ }^{1}$. El objetivo consistió, en aquella ocasión, en el estudio de las implicaciones que el sistema de horarios y actividades del trabajo productivo (volumen, ritmos, turnos, etc.) tiene sobre la distribución de las actividades del trabajo reproductivo y las del tiempo libre entre los miembros del hogar, básicamente entre hombres y mujeres. En dicha empresa, la negociación del tiempo de trabajo constituía uno de los temas controvertidos en la negociación de los convenios, siempre dentro de una lógica de intercambio de flexibilidad temporal por estabilidad laboral. El objetivo de dicha investigación general no se centró tanto en el análisis de cómo la modificación del tiempo de trabajo, consecuencia de la negociación de los convenios, repercutía sobre la relación entre las actividades y los tiempos del trabajo productivo y reproductivo, dado que tal modificación no era lo suficientemente importante; nuestro estudio se centró en el análisis de cómo las diferencias del tiempo y las actividades de trabajo de tres secciones elegidas ${ }^{2}$ de la empresa influían en

1. Esta investigación la llevó a cabo el equipo QUIT en una empresa multinacional de producción de medios de comunicación audiovisual. La investigación fue financiada por la DGICYT (SEC98-0570).

2. Tipología de sistemas horarios en la empresa estudiada

$\begin{array}{lllll} & \text { Tipo 1 } & \text { Tipo 2 } & \text { Tipo 3 } & \text { Tipo 4 } \\ \text { Sección: } & \text { Inserción } & \text { Producción en cadena } & \text { Producción en cadena } & \text { Diseño } \\ \text { Horario: } & \text { Rotatorio } & \text { Turno de mañana } & \text { Turno de tarde } & \text { Horario partido } \\ \text { Horas/semana: } & \text { 40 o menos } & 40 \text { o menos } & \text { 40 horas } & \text { Más de 40 }\end{array}$

El tipo 1 corresponde a la sección de Inserción, que marca de alguna manera el volumen y la dinámica de la producción y donde más directamente se aplican las condiciones de la producción just in time. Por otro lado, es donde más ha invertido la empresa en automatización y donde hay mayor necesidad de amortización. Todo ello hace que sea «el lugar» donde mayor implantación tiene la flexibilidad temporal, en concreto, por turnos de $8 \times 3$, los siete días de la semana con cambios rotativos quincenales y donde son mayores los cambios de planificación en la distribución anual del trabajo según la demanda. Los tipos 2 y 3 corresponden a las secciones de Producción, en las que prima como horario y sistema de trabajo el modelo más tradicional de la producción taylorista: cadena serializada, manual y rígida. Hay dos turnos de horario continuado, uno de mañana y uno de tarde; no hay rotación ni se trabaja durante los fines de semana. De hecho, es la sección y el horario que más facilita la compatibilidad del trabajo en la empresa con otros tipos de dedicación, por ejemplo: el trabajo doméstico-familiar. Se ha diferenciado entre los dos horarios dadas las características de las personas que trabajan en dichos turnos y las repercusiones diferenciales que dichos turnos tienen en el conjunto de la vida de los trabajadores y las trabajadoras. En los períodos de fuerte deman$\mathrm{da}$, se incorporan a estas secciones trabajadores y trabajadoras con contratos temporales de estación. El tipo 4 corresponde a la sección de Diseño, que es la unidad de creación y desarrollo tecnológico de la empresa y donde se trabaja en equipo. Se trabajan ocho horas diarias en un sistema de horario partido, pero pueden ser ampliadas diariamente, semanalmente e incluso en vacaciones. Se trata de una flexibilidad temporal elástica y extensiva, muy determinada por las exigencias de la empresa, aunque normalmente no se trabaja durante el fin de semana. Es también la sección donde se cuenta con instrumentos más informatizados e inteligentes. Esta sección tiene un modelo de horario clásico, aunque con condiciones que pudieran favorecer la aparición de alguna posibilidad de autogestión temporal, pero siempre en la dirección de una mayor ampliación: diaria, semanal y a veces en semanas de vacaciones. 
el ámbito reproductivo y del tiempo libre. Estas diferencias se revelaban lo suficientemente significativas en la intensidad y en los ritmos de las actividades y el tiempo laboral y en su organización productiva, como para influir en los tiempos, las actividades y la responsabilidad del hogar y/o la familia y del tiempo libre. Además, dichas secciones establecían una clara diferenciación por género (nula presencia de las mujeres en Inserción, mínima en Diseño y máxima en Producción) y por otras características y condiciones profesionales y sociofamiliares, que fueron consideradas siguiendo diversas orientaciones teóricas de la investigación ${ }^{3}$.

El análisis de los resultados de aquella investigación puso de manifiesto que las diferencias de actividades y horarios entre las secciones estudiadas (con una fuerte desigualdad en la distribución de hombres y mujeres en ellas y, por tanto, de la formación, cualificación, prestigio profesional, responsabilidad y retribución) constituían también un factor o un condicionante importante en el reparto no equitativo, entre hombres y mujeres, de las tareas y las responsabilidades domésticas, entendidas en sentido amplio. El análisis puso también de relieve que, además de las condiciones productivas, hay otras concomitantes decisivas en dicho reparto desigual, como son las condiciones sociofamiliares y la "cultura heredada", con gran poder sobre los modos y las pautas de acción y sobre las opiniones y las actitudes acerca de la distribución de las tareas del hogar.

El análisis de la organización temporal de la vida cotidiana de la plantilla de trabajadores y trabajadoras de la empresa estudiada, como caso ilustrativo de un fenómeno generalizado, constataba la perdurabilidad de una desigualdad que, por razón de género, conduce a la reproducción de dos modelos dominantes fundamentales: el de las mujeres con doble presencia que sincronizan la vida laboral y la familiar, y el de los hombres desincronizados y centrados en su vida laboral. Pero, además, ésta es una situación que responde también a una política de contratación segregadora de la plantilla por parte de la empresa por

3. Las orientaciones teóricas que constituyeron el marco conceptual y la guía interpretativa en dicha investigación (relaciones, vínculos y/o dependencias mutuos, entre los ámbitos productivo, reproductivo y del tiempo libre) se referían, primero, a la sociología de la vida cotidiana, de los modos de vida y del tiempo social en tanto que conceptos y/o perspectivas englobantes y/o transversales a dichos ámbitos, y, segundo, a los modelos de flexibilidad y centralidad productiva, reproductiva y/o de vida (Lozares, López y Domínguez, 1998; Lozares y Verd, 2004; Lozares, López y Martí, 2004; P. A. Adler, P. Adler y Fontana, 1987; Bertaux, 1983; Bertaux-Wiame, 1983; Elias, 1985; Séller, 1977; Javeau, 1980, 1983, 1984; Lefebvre, 1975, 1981; Maffesoli, 1989; Mendizábal, 1984; Remy, Vove y Servais, 1978; Salvador Juan, 1995; Wodz, 1987; Zerubavel, 1981, 1987; Balbo, 1991; Belloni, 1986; Borghi y La Rosa, 1996; Boulin y otros, 1998; Carrasquer, Torns, Tejero y Romero, 1998; Gasparini, 1998; Grossin, 1974; Torns, 1994). Los métodos de recogida de información y análisis se correspondieron con la naturaleza cuantitativa y de exploración de los discursos de las personas y de los grupos de los cuatro tipos señalados en la nota 2 precedente; esto es, una conjugación de encuestas a trabajadores y trabajadoras de las tres secciones, entrevistas informativas y semidirigidas y grupos de discusión (Lozares, Verd, 2004; Lozares, López, Martí, 2004). 
razón de género y en la que se simultanean formas de organización temporal de la vida cotidiana con una división sexual y técnica del trabajo que en nada favorecen el cambio hacia la conciliación. El objetivo de este artículo consiste en mostrar dichos resultados a partir de la explotación de una encuesta realizada en la empresa. En primer lugar, se presentará el planteamiento del estudio y el concepto de conciliación para, a continuación, explicitar la metodología seguida en el análisis y los resultados obtenidos.

\section{Cambios, planteamientos y supuestos}

\section{Los cambios y la situación}

La desigual distribución, entre hombres y mujeres, de las actividades y de las tareas y las responsabilidades domésticas y familiares depende, evidentemente, de las condiciones y de los ritmos de los tiempos y las actividades productivas, y también, como trataremos de mostrar en este artículo, de las condiciones sociofamiliares. Pero ambas condiciones y sus resultados se enmarcan y están influidos también por un conjunto de factores socioeconómicos, profesionales, demográficos, culturales e ideológicos que perpetúan o modifican, más o menos fuertemente según la dirección de los cambios en dichos factores, la distribución equitativa de las tareas, actividades y responsabilidades del hogar entre hombres y mujeres. Dicha influencia depende también de la cultura de las capas o los sectores sociales en los que se inscriben tales factores. Pero los cambios hacia una orientación más equitativa, ni son lo suficientemente extensos y progresivos, ni están asegurados como irreversibles: la cultura y la historia sociales heredadas tienen un peso considerable sobre una visión y una práctica por las que las tareas y responsabilidades del hogar son de atribución exclusiva de la mujer, como si ello fuera «lo más natural del mundo». Pero también se ha de afirmar que estos cambios han ido desvelando y puesto de manifiesto las desigualdades que se producen en la realización de dichas actividades por la vía de su crítica y reivindicación igualitaria, y han comenzado tímidamente a resolverlas por medio de su externalización, sea por la asalarización, sea por ser asumidas parcial y ocasionalmente por las políticas públicas.

Los cambios están también generando necesidades sociales diferentes con respecto al hogar y al cuidado de las personas que exigen más y nuevas dedicaciones, con el riesgo y el hecho de resolverse de la manera tradicional. Si a ello añadimos el positivo aumento, progresivo, importante y suponemos que imparable, en estas décadas, de la presencia de la mujer en las actividades productivas remuneradas y que dicha presencia e implicación no se ha visto correspondida, al menos de manera importante, con una mayor dedicación de otros miembros del hogar, más concretamente de los hombres, a las tareas domésticas, entonces, la necesidad de una conciliación o "pacto de género» es más acuciante que nunca. Ni las políticas públicas ni las empresas facilitan de manera importante el desencallar todo el cúmulo de dificultades para la necesaria conciliación. 
Los resultados siguen evidenciando que las mujeres que ejercen un trabajo productivo y remunerado no llegan a conciliar con su pareja la distribución equitativa de las tareas domésticas, de tal manera que acumulan mayor carga total de trabajo, tienen menos tiempo libre, siendo al mismo tiempo conscientes de la necesidad de un tiempo para ellas mismas, del trabajo externo y productivo que las emancipa de otras dependencias y les confiere un tipo de personalidad. En una encuesta reciente, para la población en general, se aseveran algunas de estas afirmaciones ${ }^{4}$.

- Sobre la dificultad de compatibilizar tiempo y actividad de trabajo fuera y dentro del hogar, el 46\% de los encuestados opinan que la compatibilización es complicada o muy complicada. Según el sexo, los porcentajes pasan del $40,6 \%$ entre los hombres al 51,2\% entre las mujeres. Entre las mujeres, estos porcentajes son del $54,6 \%$ para las ocupadas, del $49 \%$ para las amas de casa y del $36,9 \%$ para las situaciones de inactividad.

- Las personas que se muestran de acuerdo con la opinión de que «las dificultades para compaginar la vida familiar y laboral deterioran la educación de los hijos e hijas» son el 63,9\% entre los hombres y del 56,8\% entre las mujeres. Las personas que se muestran de acuerdo con la opinión «el uso de medidas de conciliación provocan conflictos en el ámbito laboral», constituyen el $51 \%$ entre los hombres y el $44 \%$ entre las mujeres.

- Sobre el total de la muestra, el tiempo medio dedicado a las tareas del hogar de los hombres es de 1 hora y 32 minutos, y de las mujeres, de 4 horas y 2 minutos. Si las personas están ocupadas, los tiempos son, respectivamente, de 1 hora y 30 minutos y de 3 horas y 10 minutos.

- Sobre la base de la población con menores dependientes, el tiempo medio dedicado al cuidado de los hijos e hijas menores por parte de los hombres es de 2 horas y 10 minutos, y de las mujeres, de 4 horas y 1 minuto. Si, además, las personas están ocupadas, los tiempos son, respectivamente, de 2 horas y 20 minutos, y de 3 horas y 38 minutos.

- Sobre la base de la población con mayores dependientes, el tiempo medio dedicado al cuidado de mayores por parte de los hombres es de $58 \mathrm{minu}-$ tos, y el de las mujeres, de 1 hora y 57 minutos. Si las personas, además, están ocupadas, los tiempos son, respectivamente, de 53 minutos y de 1 hora y 36 minutos.

4. Encuesta del Observatorio para la Igualdad de Oportunidades entre Mujeres y Hombres (Madrid, 5 de mayo de 2005), realizada por GPI Consultores dentro de Estudio sobre la conciliación de la vida familiar y la vida laboral: situación, necesidades y demandas. El estudio consiste en un análisis de fuentes documentales y estadísticas, entrevistas con agentes socioeconómicos y personas expertas y grupos de discusión. Concretamente, la encuesta fue de una muestra de 4.000 personas de ambos sexos y en edad laboral. 


\section{Un concepto amplio de la conciliación e hipótesis}

El concepto de conciliación no es simple, ya que no puede reducirse ni a las políticas públicas sobre dicha problemática, ni a la negociación en la empresa entre agentes, ni a un acuerdo más o menos explícito entre las personas, en particular hombre y mujer, dentro del hogar. Dadas las condiciones y los contextos que enmarcan la desigualdad en la distribución de las tareas domésticas, los factores que influyen en dicha desigualdad, los agentes en juego y las consecuencias que genera la conciliación, ha de contemplarse más bien como un concepto, si no complejo, al menos jalonado y atravesado por diversos componentes y dimensiones que conviene tener presentes; de lo contrario, se puede dar del mismo una visión y un análisis parciales.

El concepto de conciliación puede contemplarse y construirse como la confluencia de dos componentes. El primero, desde la personalidad social del individuo y consiste en el equilibrio ponderado que cada persona y, en este caso concreto, cada miembro del hogar establece en la distribución "conciliada» de sus actividades, tiempos, tareas, responsabilidades entre las centralidades de su trabajo productivo, reproductivo y del resto de las actividades y tiempos. El segundo, desde el colectivo hogar para las personas que a él pertenecen y consiste en la distribución equitativa y «negociada», explícita o implícitamente, entre sus miembros, y más concretamente entre los hombres y las mujeres, de dichas actividades, tareas y responsabilidades.

Los componentes contextuales de la conciliación se consideran también, desde nuestra acepción, parte integrante de su definición, ya que actúan o interfieren, facilitando o dificultando la conciliación precedente. En este sentido, se pueden diferenciar varios niveles contextuales. El más cercano se configura a partir de los ámbitos de la vida cotidiana de las personas, su trabajo y la vida del hogar, esto es: 1) el de las condiciones socioproductivas (horarios, actividades y características de los trabajadores y las trabajadoras) y 2) el de las condiciones sociofamilares. Ambos contextos influyen decisivamente sobre la distribución desigual de las tareas y responsabilidades domésticas, condicionando de manera determinante la conciliación. Es solamente sobre este aspecto contextual que versa este artículo. Otros contextos más generales se refieren a las políticas de las empresas, a los agentes sindicales, a las negociaciones correspondientes, a las políticas públicas en cualquier nivel de la Administración y, más globalmente, a los estados de opinión, representaciones, creencias, cultura o ideología, así como a las normas y a los hábitos más o menos explícitos o dados por supuestos.

Nuestro objeto de estudio no consiste en analizar todos los aspectos enunciados que componen esta constelación de la conciliación. Muchos de ellos han sido ya tratados certera y suficientemente: Torns, Carrasquer, Borràs y Roca (2002) y Bimbi (1999), que analizan las dificultades y las resistencias socioculturales a la conciliación; Brullet (2000) y Escobedo (2000), sobre aspectos vinculados a la conciliación; CE (1999), Valiente (2000), Carrasco, Alabart, Mayordomo y Montagut (1998), Chinchilla y Poelmans (2002), Junter-Loiseau y Tobler (1999) y Torns (2004), sobre políticas públicas. 
En este artículo, que retoma algunos de los datos de la investigación anunciada precedentemente, más concretamente los de la encuesta, se tratan específicamente las condiciones socioproductivas (horarios, actividades y características de los trabajadores y las trabajadoras) y las sociofamiliares y sobre todo la conjunción de ambas, como factores que influyen de manera determinante sobre la distribución desigual de las tareas, las prácticas y las responsabilidades domésticas. La hipótesis que proponemos consiste en mostrar que ambas condiciones, concomitantemente, poseen tal divergencia en sus pautas y exigencias de conducta sociotemporal, esto es, funcionan tan independientemente en las exigencias de dedicación y responsabilidad, que ello dificulta los procesos y las situaciones de conciliación temporal, concretamente entre hombres y mujeres en tanto que pareja en el hogar. Del conjunto desplegado antes sobre el concepto de "conciliación", sólo nos centramos aquí en los «contextos de la conciliación» inmediatos, esto es, en la relación entre: 1) las condiciones y características socioproductivas (horarios, actividades y características de los trabajadores y las trabajadoras) y 2) las sociofamiliares como origen conjunto de las dificultades de la conciliación. Dicha relación define un espacio social que interviene directamente en la distribución, entre hombres y mujeres, de las actividades, tareas, organización y responsabilidades del hogar y la familia y, por tanto, que opera muy directamente sobre las condiciones de la conciliación, lo que constituye un campo potencial de dificultades individuales y de la pareja para la conciliación.

\section{Metodología}

El análisis que aquí presentamos se basa, como venimos insistiendo, solamente en los datos de la encuesta llevada a cabo en la empresa a partir de un cuestionario administrado a 260 trabajadores y trabajadoras de las secciones de producción, inserción y diseño, sobre una plantilla de 1.200 trabajadores ${ }^{5}$. En el proceso de análisis actual, hemos ido más allá del método de análisis bivariado, recurriendo al multivariado, concretamente, al análisis de correspondencias múltiples (ACM) y al análisis de clasificación automática (ACL). El tratamiento ha consistido en la dimensionalización y tipificación, pero realizado parcialmente por ámbitos y superponiéndolos progresivamente hasta llegar a la tipología general de las variables y de las relaciones entre los ámbitos productivos, reproductivos y de condiciones sociofamiliares ${ }^{6}$. De dicha superposición sucesiva, solamente se analizan aquí las acumulaciones de varianza de los diferentes ACM realizados; el análisis de factores y de clasificación automática se examina para el conjunto de todos los ámbitos y condiciones 1, 2 y 3 de la tabla 1.

5. Para un análisis descriptivo bivariado, ver Lozares y Verd (2004), y Lozares, López y Martí (2004).

6. Sobre el proceso de construcción tipológica a través de métodos multivariables, se puede consultar C. Lozares y otros (1998), y P. López (1996). 
Tabla 1. Ámbitos y variables del análisis.

\section{1. Ámbito del trabajo productivo en la empresa}

a) Variables temporales: sistema del horario en la empresa, edad y tiempo en la empresa Horario de trabajo: partido, rotatorio, mañana o tarde.

Antigüedad en la empresa: 1974-92, 1993-97 o 1998-2000.

Edad del trabajador o la trabajadora: 18-24, 25-29 o 30-45.

b) Variables que caracterizan el ámbito productivo

Sección de la empresa: Diseño, Inserción o Producción.

Categoría profesional: técnico superior o medio, operario o administrativo.

Nivel de estudios: primarios-EGB, BUP, FP1, FP2 o universitarios.

Tipo de contrato: indefinido o temporal.

Género: varón o mujer.

\section{Condiciones sociofamiliares}

a) Variables que definen el tiempo de ciclo de vida como expresión de una temporalidad socio-familiar

Tipo de convivencia: vive con los padres, vive solo, en pareja, pareja con hijos u otras.

Presencia de personas dependientes: ninguna, personas mayores, hijos.

b) Variables temporales familiares vinculadas al trabajo productivo

Personas ocupadas: ocupado él-ella, pareja ocupada, él-ella + padres u otras situaciones.

Coincidencia horario de trabajo: coinciden horarios en la pareja, parcialmente, no coinciden o sin pareja.

\section{3. Ámbito del trabajo reproductivo}

a) Percepción de las dificultades del trabajo reproductivo

Tareas del hogar: dificultades para realizarlas o sin dificultades.

Cuidado de los hijos: con dificultades para realizarlo o sin dificultades.

b) Reparto del trabajo reproductivo

Quién cocina: él o ella, ambos, él o ella + pareja, él o ella + padres, otras situaciones. Quién limpia: él o ella, ambos, él o ella + pareja, él o ella + padres, otras situaciones.

Quién compra: él o ella, ambos, él o ella + pareja, él o ella + padres, otras situaciones.

Quién cuida a los hijos: él o ella, él o ella + pareja, él o ella + padres, otras situaciones, sin hijos.

Quién hace el bricolaje: él o ella, ambos, él o ella + pareja, él o ella + padres, otras situaciones.

Además, nos hemos interesado en comprobar la localización y el rol que determinadas variables ocupan y desempeñan en el espacio de dichas condiciones socioprofesionales y sociofamiliares, como son el horario de la empresa con sus marcadas diferencias: producción de mañana (continuo), producción de tarde (continuo), inserción (rotatorio), y diseño (partido) y género. 


\section{Los ámbitos estudiados, las variables y el proceso de investigación}

Los grupos de ámbitos y variables manejados son los siguientes ${ }^{7}$ :

1. Ámbito del trabajo productivo en la empresa: tipos de horarios, antigüedad en la empresa, edad, secciones consideradas en la empresa, características profesionales y género.

2. Condiciones sociofamiliares: tipo de convivencia, presencia de personas dependientes, personas ocupadas y coincidencia de horarios.

3. Ámbito del trabajo reproductivo: percepción de las dificultades del trabajo reproductivo y reparto del trabajo reproductivo.

\section{Análisis}

Veamos ahora qué es lo que sucede como resultado de interrelacionar los ámbitos y las condiciones entre sí: trabajo productivo en la empresa, las condiciones sociofamiliares y el reparto y las dificultades del trabajo reproductivo. Nos fijaremos en particular en las varianzas acumuladas por los factores de cada análisis de dimensionalización (ACM), en la propia dimensionalización global y en la tipificación que se obtiene (ACL) ${ }^{8}$.

\section{Las varianzas acumuladas}

El análisis del comportamiento de la varianza acumulada se hace para los ámbitos y para las sucesivas incorporaciones o interrelaciones de unos ámbitos con otros. La tabla 2 es elocuente de por sí; en ella se puede contemplar la capacidad de una mayor o menor estructuración (o dispersión) de cada ámbito y de las superposiciones de otros en los tres primeros ejes. Se sobreentiende que si la acumulación de dos o más ámbitos obtiene menor varianza acumulada en los tres ejes que la que tenía cada uno de los ámbitos separadamente en los dichos ejes, la conjunción de los ámbitos procura un espacio social menos estructurado o más disperso (esto es, los ámbitos están conjuntamente menos asociados de lo que lo estaba cada uno), lo que nos llevaría a la conclusión de que cada ámbito opera de forma más independiente. Volveremos a ello en los primeros apartados del punto 4 («Interpretación y conclusiones»).

\section{La dimensionalización}

En el análisis de dimensionalización, se han considerado cinco factores principales ${ }^{9}$. Se identifica la primera dimensión en términos de formas de convivencia, tipo de ocupación familiar, tipo de familia y ocupación familiar, esto es, por las condiciones sociofamiliares. A ellas se acoplan también la edad y la

7. En la tabla 1 se detallan las categorías correspondientes.

8. Los análisis se han efectuado con el programa estadístico SPAD, versión 5 .

9. En el gráfico 1 se representan los dos primeros y centrales. 
Tabla 2. Varianza acumulada por los tres primeros ejes en los análisis de correspondencias.

\begin{tabular}{llll}
\hline Varianza acumulada por el: & Eje 1 & Eje 2 & Eje 3 \\
\hline 1a. Sistema de horarios y temporal & $25 \%$ & $43 \%$ & $59 \%$ \\
2. Condiciones sociofamiliares: $2 \mathrm{a}+2 \mathrm{~b}$ & $26 \%$ & $39 \%$ & $52 \%$ \\
$1 \mathrm{a}+2$ & $20 \%$ & $30 \%$ & $39 \%$ \\
2. Condiciones sociofamiliares & $26 \%$ & $39 \%$ & $52 \%$ \\
1b. Ámbito productivo + género & $20 \%$ & $36 \%$ & $54 \%$ \\
$\quad 1 \mathrm{~b}+2$ & $15 \%$ & $27 \%$ & $35 \%$ \\
3. Ámbito reproductivo & $18 \%$ & $28 \%$ & $36 \%$ \\
Ámbito reproductivo + género y edad & $17 \%$ & $27 \%$ & $35 \%$ \\
3. Ámbito reproductivo & $18 \%$ & $28 \%$ & $36 \%$ \\
1b. Ámbito productivo & $25 \%$ & $48 \%$ & $61 \%$ \\
$\quad 1 b+3$ & $12 \%$ & $22 \%$ & $29 \%$ \\
2. Condiciones sociofamiliares & $26 \%$ & $39 \%$ & $52 \%$ \\
Ámbito reproductivo + ámbito productivo: 3+ 1b & $12 \%$ & $22 \%$ & $29 \%$ \\
$\quad$ lb + 2 + 3 & $14 \%$ & $22 \%$ & $28 \%$ \\
1a. Sistema de horarios y temporal & $25 \%$ & $43 \%$ & $59 \%$ \\
1b. Ámbito productivo (con género) & $20 \%$ & $36 \%$ & $54 \%$ \\
2. Condiciones sociofamiliares & $26 \%$ & $39 \%$ & $52 \%$ \\
3. Ámbito reproductivo & $18 \%$ & $28 \%$ & $36 \%$ \\
Interrelación de los ámbitos: $1 \mathrm{a}+1 \mathrm{~b}+2+3$ & $13 \%$ & $23 \%$ & $29 \%$ \\
\hline
\end{tabular}

antigüedad, acentuando una connotación propia a la temporalidad del ciclo de familia. De esta forma, en un polo, se sitúan los trabajadores y las trabajadoras de más edad y antigüedad en la empresa, que viven en pareja (con o sin hijos) y reparten el trabajo reproductivo entre sus miembros de formas diversas. En el otro polo se sitúan los trabajadores y las trabajadoras que viven solos, en otras situaciones, y, en la parte más extrema, aquéllos y aquéllas que viven con los padres. En este último caso, son los padres, en general ocupados, quienes asumen principalmente el trabajo reproductivo.

La segunda dimensión se identifica a partir del sistema de horarios, secciones y otras características socioprofesionales de los trabajadores y las trabajadoras de la empresa, así como por la existencia de dificultades para realizar el trabajo reproductivo. De alguna manera, sobre esta dimensión de división de trabajo y sistema de horarios se superpone la compatibilidad o dificultad de sincronía o no con relación a la posibilidad de compaginar el trabajo reproductivo. Así, en un extremo, se sitúan los trabajadores, varones, de la sección de diseño de cualificaciones y estudios altos y con horario partido próximos a los de inserción con estudios y cualificaciones medias y con horario rotatorio, que manifiestan dificultades para realizar las actividades del trabajo doméstico o el cuidado de los hijos o de personas dependientes, si los tienen. Si viven en pareja, manifiestan una cierta tendencia a coincidir su jornada con la de su pareja, afirman que las tareas de cocinar y de comprar las realizan ambos miem- 


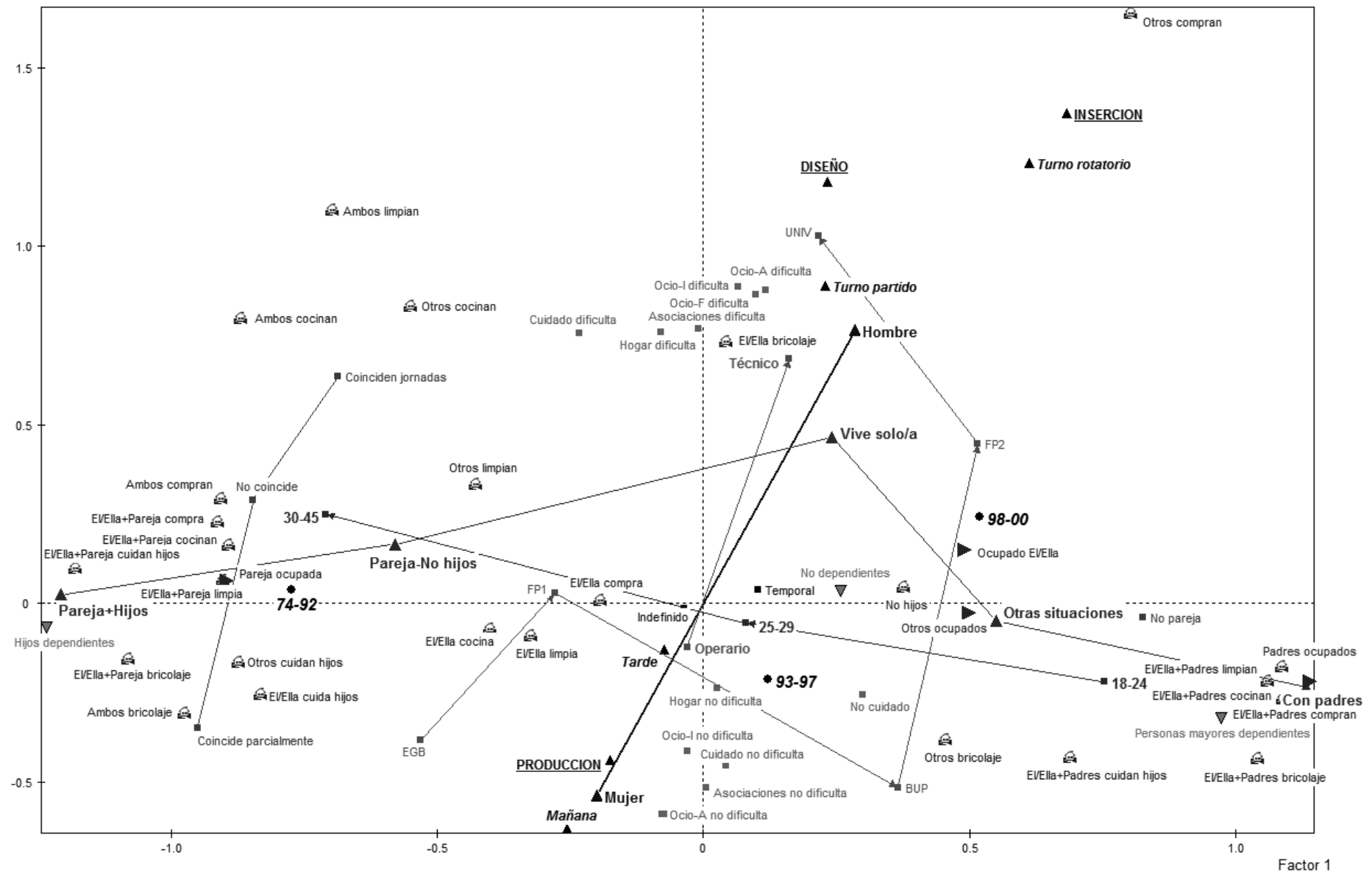

Gráfico 1. Análisis global: trabajo reproductivo, reproductivo, condiciones sociofamiliares y sistema de horarios. 
bros u otras personas; para los hombres, siempre el bricolaje. Su situación es de desincronización con el trabajo doméstico y de centralidad en el trabajo productivo. Frente a ellos, en el otro extremo, se sitúa un perfil que identifica a las mujeres de producción, en turnos de mañana o tarde, con el menor nivel de cualificación y formativo, que manifiestan no tener dificultades para asumir el trabajo reproductivo y el cuidado de personas dependientes, mostrando por lo general coincidencias parciales de jornada con sus parejas. El tiempo de trabajo y de no trabajo permiten compatibilizar los dos tipos de trabajo, el productivo y el reproductivo, y hacer patente la situación de doble presencia (Balbo, 1994; Carrasquer y Torns, 2002). Las mujeres concilian esta doble presencia en relojes imposibles, los hombres la desincronizan con su centralidad productiva.

Una tercera dimensión matiza la anterior para destacar las diferencias derivadas de la categoría profesional y el nivel de estudios que tienen las distintas secciones de la empresa; se trata de una dimensión netamente productiva que refleja las diferencias en la estructura ocupacional de la empresa, para sobresaltar principalmente el perfil específico de los trabajadores más cualificados y formados de la sección de diseño.

La cuarta dimensión pone de manifiesto la especificidad de las personas que viven solas frente al resto, destacando la situación en la que han de asumir el trabajo reproductivo de cocinar, limpiar y comprar. Por último, la quinta dimensión contrapone las parejas sin hijos frente a las parejas con hijos.

\section{La tipificación}

La tipificación se construye en base a esta estructuración de relaciones entre los diferentes ámbitos que configuran las actividades y el tiempo de trabajo, productivo o reproductivo, junto a las características sociofamiliares. A través del ACL, se obtiene la tipología global, cuyos rasgos descriptivos más destacados son los siguientes (ver gràfico 2).

Tipo 1 ( $8 \%$ de la plantilla). Se trata de un grupo formado únicamente por trabajadores de la sección de diseño con horario partido. En su mayoría, son hombres $(85 \%)$ de edades comprendidas entre los 25 y los 45 años, con un alto nivel educativo ( $81 \%$ con estudios universitarios) y la categoría profesional de técnico $(81 \%)$. No se trata de los más antiguos de la plantilla, ya que la mitad de ellos entró en los últimos años. Se observan diversas formas de convivencia en su interior, si bien sobresale que se trata de personas que viven en pareja y sin hijos (34\%) o bien con los padres (32\%), y donde apenas se dan situaciones de personas dependientes. El horario partido conlleva un alto nivel de coincidencia de horarios con la pareja (que suele también trabajar) y una tendencia a manifestar dificultades para ocuparse del trabajo reproductivo. En consecuencia, destaca, con relación al resto, un relativo compartir las tareas domésticas con la pareja o los padres (cocinar, limpiar y comprar), pero también asalariándolas a otras personas (caso de la limpieza). La excepción es el 


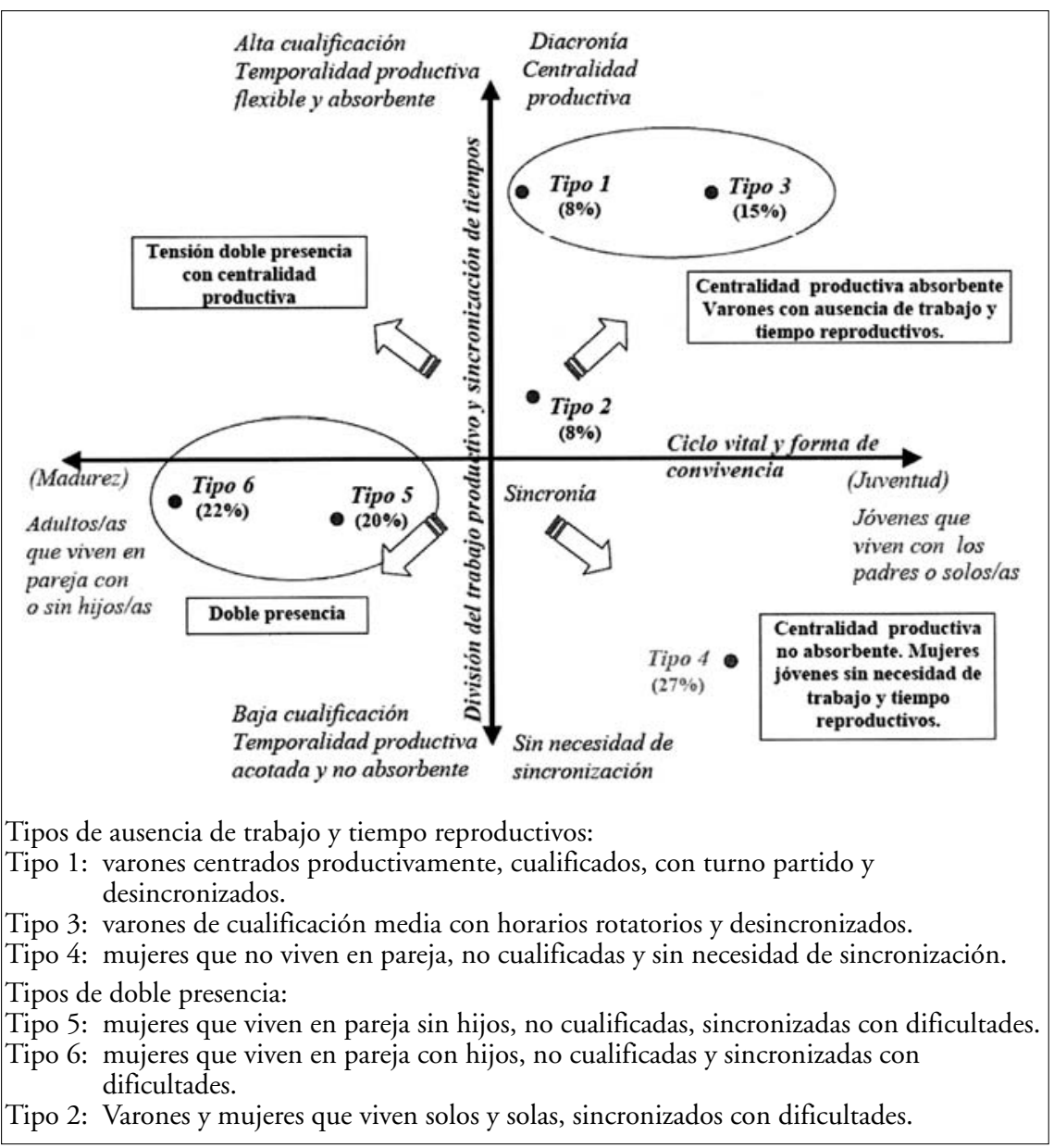

Gráfico 2. Dimensionalización y tipología global de los tiempos.

bricolaje, mayoritariamente asumido por los hombres y, en mucha menor medida, la compra. Por tanto, se trata de un grupo absorbido por el trabajo productivo, que asume poco el trabajo reproductivo y que manifiesta dificultades para sincronizar el tiempo laboral con el familiar y de ocio. Se trata, en resumen, de varones cualificados de diseño, centrados productivamente, con turno partido y desincronizados.

Tipo 2 ( $8 \%$ de la plantilla). El rasgo más sobresaliente de este grupo es que, en su mayoría, son personas que viven solas $(75 \%)$, aunque hay una parte $(25 \%)$ que se encuentra en otras situaciones de convivencia; pero en ningún caso se dan parejas ni existen personas dependientes. Están presentes todos los gru- 
pos de edad, aunque destaca el de 30-45 años (45\%), que son los mayores en la empresa, dada la edad de los trabajadores y las trabajadoras, y hay tanto hombres como mujeres. Desde el punto de vista productivo, hay tanto técnicos $(25 \%)$ como operarios (75\%), destacando el nivel medio de formación de FP2 (41\%) y BUP (31\%). Aunque presentes en las distintas secciones y horarios, predomina la sección de producción y horarios de mañana y tarde. Este grupo manifiesta que realiza las tareas domésticas y que no tiene dificultades para llevarlas a cabo. En síntesis, son varones y mujeres de diferentes secciones, de cualificación y formación media, que viven solos y sin problemas de sincronización.

Tipo 3 (15\% de la plantilla). Es un grupo definido netamente por sus rasgos productivos: trabajadores de inserción en turno rotatorio (90\%), todos ellos varones, con niveles formativos de FP. Se trata de la plantilla más joven de las tres secciones. En cuanto a la convivencia familiar, viven con sus padres $(50 \%)$, en otras situaciones (19\%) o en pareja pero sin hijos (20\%). Es el grupo que manifiesta más claramente tener dificultades para realizar las tareas del trabajo doméstico, aunque lleven a cabo tareas de bricolaje y declaren que comparten tareas del hogar con sus padres o con la pareja. Por tanto, es el tipo que, junto al tipo 1 , muestra un mayor grado de desincronización entre los tiempos de trabajo y los otros tiempos. Su juventud, género y situación de convivencia implica socialmente menores obligaciones y responsabilidades con respecto al trabajo de la reproducción. Es resumen, se trata pues de varones de inserción con cualificación media, con horarios rotatorios y desincronizados.

Tipo 4 (27\% de la plantilla). Uno de los rasgos definitorios de este grupo es la forma de convivencia; o bien son jóvenes que viven con sus padres $(69 \%)$ o, en menor medida, en otras formas de convivencia pero no en pareja $(29 \%)$. Además, no hay personas dependientes en el hogar. Las distintas tareas reproductivas son compartidas o realizadas sobre todo por los padres. Es el grupo que manifiesta tener menos dificultades para realizar las tareas del trabajo reproductivo. En su gran mayoría, son trabajadoras (el $75 \%$ son mujeres) de producción, en turno de mañana (59\%) y menos en el de tarde (28\%). Por tanto, la categoría profesional característica es la de operario u operaria $(91 \%)$ y sus niveles de estudios son medios o bajos. A diferencia del grupo anterior, masculino y joven, que manifiesta problemas de sincronización favorecido por el tipo de horario, en este caso no se manifiestan, en parte gracias a la también menor carga reproductiva que deben asumir, favoreciendo así la sincronización entre tiempos de trabajo y no trabajo. En síntesis, son mujeres jóvenes que trabajan en la sección de producción poco cualificadas, que viven con los padres y sin problemas de sincronización.

Tipo 5 (20\% de la plantilla). Se trata también de un grupo mayoritariamente de mujeres $(80 \%)$. Todas trabajan en la sección de producción. Coincide también con el tipo anterior en el predominio de la categoría de operario (92\%), pero difiere en su nivel de estudios y de cualificación más bajos y en que la 
edad es algo mayor. Hay una proporción más elevada que trabaja de horario de tarde (50\%). También, a diferencia del grupo anterior, su rasgo distintivo es la forma de convivencia, el $92 \%$ vive en pareja sin hijos, ambos miembros están ocupados, pero coinciden en horarios de la jornada, sea parcial (57\%) sea totalmente $(15 \%)$. No hay personas dependientes y no manifiestan tener dificultades para realizar el trabajo doméstico (cocinar, comprar y limpiar), pero son las mujeres las que lo realizan mayoritariamente y en menor medida con la ayuda de la pareja. En consecuencia, la mayor carga reproductiva de este grupo implica un modelo de doble presencia y de sincronización con el tiempo y el trabajo productivos. El tipo está, pues, compuesto sobre todo por mujeres jóvenes con doble presencia, trabajando en la sección de producción, no cualificadas y que viven en pareja sin hijos y sincronizadas.

Tipo 6 (22\% de la plantilla). El último grupo coincide de nuevo con los dos anteriores, por ser personal operario de la sección de producción, con predominio de mujeres (76\%) y nivel de estudios medios, pero sobre todo bajos. Es el grupo de mayor edad y antigüedad en la empresa. Hay un claro predominio del horario de mañana $(76 \%)$ frente al de tarde. Su rasgo distintivo con relación a los dos precedentes es el de convivir en pareja y con hijos. Ambos están ocupados, pero el grado de coincidencia horaria en la jornada es sobre todo parcial $(47 \%)$ y, en menor medida, total (10\%). Como en el caso anterior, el trabajo doméstico lo realiza sobre todo la entrevistada, aunque se afirma que con la colaboración de la pareja. En este caso, el trabajo reproductivo conlleva la carga añadida de la atención de los hijos; tarea que recae principalmente o asume exclusivamente la entrevistada, aunque también declare que la pareja la comparte o colaboran otras personas de fuera del hogar. Manifiestan que tienen más dificultades que el grupo anterior para realizar el trabajo doméstico y atender a los hijos. Se trata, por tanto, de un grupo de mujeres con mayor carga reproductiva, con horarios laborales que facilitan la sincronización, pero con dificultades para asumir ambos trabajos. En síntesis, son mujeres adultas con doble presencia, que trabajan en la sección de producción, no cualificadas, que viven en pareja, con hijos y sincronizadas.

\section{Interpretación y conclusiones}

\section{Los ámbitos del tiempo y del trabajo productivo, reproductivo $y$ las condiciones sociofamiliares}

En el ámbito productivo, las características socioprofesionales dependen considerablemente de las secciones estudiadas; por contra, el tipo de contrato laboral es, en gran medida, independiente de las secciones. También el género está muy vinculado a las secciones y en general a los horarios del sistema productivo, idénticamente las variables de edad, antigüedad y sobre todo horarios y turnos. La variable sección posee una elevada capacidad de articulación de las variables socioprofesionales, género y el sistema de horarios, si se consi- 
dera a partir del análisis de la varianza explicada en los tres primeros ejes del ACM.

Las variables de condiciones sociofamiliares poseen también en sí un gran poder estructurador, incluso cuando se introduce el género.

El ámbito reproductivo (quién hace qué y qué dificultades se perciben para hacerlo) posee una configuración menos estructurada en los tres primeros ejes que los ámbitos precedentes. De hecho, esta mayor dispersión se debe a que el conjunto de variables que expresa el grado de dificultad para realizar tareas de hogar y el que expresa cómo se distribuye realmente el trabajo del hogar, no van muy acoplados; las primeras variables se asocian más a la situación familiar y las segundas, al sistema de horarios de la empresa y a las características socioproductivas, lo que no deja de ser sintomático de la contradicción entre los dos dominios. Esta relativamente floja estructuración no se modifica cuando se introducen las variables de género y edad; es decir, que se acoplan relativamente bien a la estructura de las variables del trabajo reproductivo.

\section{Las interrelaciones entre ámbitos}

$\mathrm{Al}$ conjugar en el mismo análisis las condiciones sociofamiliares y el sistema de horarios (muy vinculado a la producción), disminuye considerablemente el poder explicativo y estructurador de los tres primeros ejes, al dar cuenta de menor varianza acumulada que en cada uno de los dos sistemas separadamente.

Idéntica conclusión se obtiene cuando se asocian las condiciones sociofamiliares con el ámbito productivo (o características socio-profesionales), introduciendo además el género.

Dado que el sistema de horarios se asocia perfectamente a las características del ámbito productivo, se concluye que las condiciones sociofamiliares, por un lado, y el sistema de horarios junto al ámbito productivo (o características socio-profesionales), por otro, tienen un grado mucho menor de asociación que cada una de las dos dimensiones lo tienen de por sí. Además, ambos espacios sociales se despliegan sobre ejes diferentes como muestra de su independencia. El género y el sistema horario, sobre todo éste último, se contextualizan o se "pegan" más al eje que se identifica con el ámbito productivo, alejándose del de las condiciones sociofamiliares. Es decir, que la variable género queda en una posición ligeramente intermedia como signo evidente de la contradicción que produce su condición de estar entre dos aguas, aunque más cercana a las condiciones del ámbito productivo. Todo ello confirma que las exigencias del hogar y las de la producción funcionan en este caso de manera independiente, como si se tratara de dos ámbitos sociales diferenciados.

De todas maneras, se ha de destacar el hecho de que dicha independencia no se produce para las cuatro variables que configuran las condiciones sociofamiliares (tipo de convivencia, presencia de personas dependientes, personas ocupadas y coincidencia de horarios en la pareja), sino que la coincidencia de horarios de la pareja se asocia más al sistema de horarios y al ámbito productivo y las otras tres, al primer eje que expresa el ciclo de vida familiar. 
La vinculación del ámbito reproductivo (percepción de las dificultades y reparto de las tareas del hogar) con las variables de género y edad no aumenta para nada la dispersión del ámbito reproductivo, incluso lo disminuye, lo que nos habla de la vinculación de la variable género con "quién hace», "qué hace» $\mathrm{y}$ "que dificultades» se perciben al realizar las tareas del hogar.

$\mathrm{Al}$ analizar conjuntamente el ámbito productivo (o características socioprofesionales) y el ámbito reproductivo, con sus características correspondientes, disminuye considerablemente el elevado grado de asociación y estructuración que mantenía en el conjunto de los tres primeros ejes en cada uno de los ámbitos debido al volumen de varianza explicada. Parece que se juntan o se superponen dos mundos diferentes, aunque no tan marcadamente como sucede cuando se asocian las condiciones sociofamiliares y el ámbito productivo. De nuevo, género y horarios tampoco encuentran una asignación o localización clara en uno u otro ámbito, aunque con alguna mayor inclinación al productivo.

Si el ámbito reproductivo vinculado al productivo (o de caracteristicas socioprofesionales) se asocia al de las condiciones sociofamiliares, el poder estructurador de los ejes se reduce considerablemente con relación al de las condiciones sociofamiliares, pero se mantiene casi idéntico al de la vinculación entre ámbito productivo y condiciones sociofamiliares. Vista esta interrelación con respecto a los ejes, observamos que el primer factor o dimensión proviene casi exclusivamente de una cierta combinación de las variables ligadas a las condiciones sociofamiliares y al ámbito reproductivo, especialmente en términos de quién realiza realmente dichas actividades. Las incidencias del ámbito productivo y de las dificultades encontradas para llevar a cabo las actividades reproductivas debido al horario de trabajo son menores en este primer eje, contrario a lo que sucede en el segundo eje, en el que tienen un peso importante. Género y horarios, si bien están algo más identificados con la dimensión productiva, también tiene una cierta proyección sobre las condiciones sociofamiliares. Las actividades vinculadas al ámbito reproductivo están más vinculadas a las condiciones sociofamiliares.

\section{La interrelación global de los ámbitos: articulación de líneas explicativas} y grupos más característicos

Como consecuencia del análisis de la varianza y de la dimensionalización, comprobamos que la interrelación de todos los ámbitos lleva a la identificación de dos dimensiones básicas. La primera se identifica con las condiciones sociofamiliares, en particular al tipo de convivencia, ocupación familiar y dependencias familiares - la coincidencia horaria está más ligada al sistema de horarios y a las características soci-profesionales. La variable género tiene un peso más limitado sobre esta dimensión. La naturaleza de las variables que más se proyectan sobre esta dimensión nos permite identificarla como una dimensión que refleja el concepto de ciclo de vida y los tipos de ocupación familiar. La segunda dimensión aparece relacionada con el ámbito productivo, donde se repro- 
duce la división del trabajo según diferentes condiciones socioprofesionales y está también muy vinculada a la posibilidad de sincronización y asincronización entre el tiempo y las actividades de los ámbitos productivo y reproductivo y sobre todo a la percepción de si el horario productivo está a la raíz de las dificultades (o no) de llevar a cabo actividades del trabajo reproductivo. También se asocia con la coincidencia completa, parcial o nula, del horario de trabajo de la pareja, esto es, que se puede suponer que está en relación con las estrategias o la negociación de la pareja en función sobre todo del cuidado de los hijos y del horario de la producción. A esta segunda dimensión se la ha denominado de la división del trabajo productivo y de sincronización versus desincronización horaria. La variable de género y sobre todo el sistema horario tienen su proyección sobre dicha dimensión.

El análisis nos lleva a constatar ya una clara diferenciación entre mujeres y hombres en la relación que se da entre el ámbito productivo y el reproductivo; esta discriminación por razón de género contiene al menos dos aspectos. Primero, los varones tienen categorías más altas, más formación, están en las secciones más "prestigiadas" y con un trabajo más cualificado; las mujeres se ubican en la situación opuesta. Segundo, los varones son los que manifiestan tener más dificultades a causa del horario de trabajo para llevar a cabo actividades de labor reproductiva y de tiempo de no-trabajo, es decir, que son los que aparentan, según su opinión, estar más desincronizados. El hecho es tanto más llamativo cuanto que son ellos los que menos realizan las actividades domésticas, a excepción del bricolaje; por el contrario, las mujeres se encuentran en la situación opuesta. ¿ Idilio de sincronización ideal de las mujeres en su vida cotidiana, presagio de equilibrios personales y razón de justificación basada en la desincronización de los hombres para justificar el no hacer nada en casa?. Lo que de hecho sucede es que, 1) la sincronización está forzada e hipotecada en el caso de las mujeres, porque se las han de arreglar para arremeter con el trabajo productivo y el reproductivo (nadie lo haría si ellas no lo hicieran), cuando además se da por supuesto que son ellas las que deben hacerlo; 2) los hombres justifican su desinhibición en el trabajo doméstico dada su centralidad en el ámbito productivo.

Los trabajadores que viven en pareja representan un modelo de centralidad productiva, donde el horario laboral partido o rotatorio refuerza la desincronización con los otros tiempos de la vida. Por ello, son los que más manifiestan la existencia de dificultades para realizar el trabajo reproductivo. Las trabajadoras que viven en pareja representan el modelo de doble jornada, donde la carga total de trabajo, productivo y reproductivo, adquiere todo su peso. El horario laboral de mañana o de tarde caracteriza la sincronización con el tiempo de la reproducción. Por ello se explica que sean las que manifiesten más mayoritariamente la ausencia de dificultades para realizar el trabajo reproductivo.

Finalmente, nos encontramos con la parte de la plantilla más joven de la empresa, distribuida entre los diferentes horarios, que mayoritariamente vive con los padres, siendo éstos quienes asumen básicamente el trabajo reproductivo. 


\section{La temporalidad social, el tiempo de la empresa}

\section{y la articulación de los ámbitos en la vida cotidiana}

Dos tipos diferentes de temporalidad social atraviesan los ámbitos, las dimensiones y los grupos estructurados de los trabajadores y las trabajadoras y secciones estudiados en la empresa.

El primer tipo de temporalidad, es del horario productivo que se vincula al ámbito productivo y a sus características socioprofesionales. Está asociado a la segunda dimensión, es decir, al ámbito productivo, pero también a la percepción de la dificultad o no de realizar actividades de reproducción según dicho horario. El análisis realizado pone de manifiesto cómo la organización socioproductiva de la empresa marca pautas y modelos asociados estrechamente con los distintos horarios laborales y secciones de la empresa, lo que se traduce en la configuración de grupos diferenciados de trabajadores y trabajadoras de la plantilla. Los horarios partidos, intensivos de mañana o de tarde y los rotatorios introducen la lógica de la organización flexible propia a la empresa. Dicha flexibilidad, elástica y modulada según los intereses de la empresa, pero implacable y absorbente para la vida del trabajador o la trabajadora, no sólo estructura rígidamente su vida cotidiana y condiciona, por tanto, cualquier posible pacto y conciliación entre los miembros del hogar, sino que interviene muy determinantemente en el conjunto de los flujos temporales, tanto de autogestión del tiempo para sí como de los otros tiempos sociales que componen la vida cotidiana de las personas.

El segundo tipo de temporalidad se vincula o corresponde a las condiciones sociofamiliares y, más extensamente, al ciclo de vida familiar, ya que se configura a partir del tipo de convivencia en el hogar (vive con los padres, solo, en pareja, en pareja con hijos u otras situaciones); la presencia de personas dependientes (ninguna, personas mayores o hijos); las personas ocupadas (ocupado sólo él o ella, pareja ocupada, él o ella + padres u otras situaciones), la edad y la antigüedad en la empresa. Como hemos visto, esta dimensión opera de manera independiente con relación a la primera.

Ambas temporalidades estructuran y condicionan la vida cotidiana de las personas de las secciones analizadas, al incidir en sus comportamientos y en la distribución de sus actividades. Por ello, dichas temporalidades, que provienen de las exigencias de la producción y su temporalidad y de las condiciones sociofamiliares y su temporalidad son mucho más que un marco descriptivo o interpretativo; se trata de factores contextuales reales que evidencian y explican, facilitando o poniendo dificultades, a la conciliación entre los miembros de la pareja del hogar.

El análisis de los datos de la encuesta no nos permite entrar en el terreno de la subjetividad, pero se pueden apuntar algunos elementos ilustrativos sobre la dedicación al tiempo de trabajo reproductivo. Se dan vivencias distintas de los tiempos cotidianos de los trabajadores y las trabajadoras, en la medida en que el trabajo y el tiempo productivo suelen ser centrales y determinantes en la organización de sus vidas y en la medida que se dan sincronías 
o desincronías en su articulación con otros ámbitos. Esta desincronización es también variable en naturaleza y presencia según la situación y las características sociales en que se encuentra cada tipo de trabajador o trabajadora, pero también y sobre todo en función del momento o la etapa de su ciclo vital, del género y de la propia percepción y el significado atribuido al propio tiempo y a su uso.

\section{Bibliografía}

Adler, P.A.; Adler, P.; Fontana, A. (1987). «Everyday life sociology». Ann. Rev. of Sociology, 13: 217-235.

Balbo, L. (1987). Time to Care. Politiche del tempo e diritti quotidiani. Milán: Franco Angeli.

- (1991). Tempi di vita. Milán: Feltrinelli.

- (1994). La doppia presenza. En: Borderías, C.; Carrasco, C.; Alemany, C. (eds.). Las mujeres y el trabajo. Barcelona: FUHEM-Icaria.

BeLlONI, M. C. (1986). «La struttura del tempo quotidiano». En: BimBi, F.; CAPECCHI, V. Strutture e strategie della vita quotidiana. Milán: Franco Agneli.

Bertaux, D. (1983). "Vie quotidienne ou modes de vie». Revue Suisse de Sociologie, 1: 69.

BERTAUX-Wiame, I. (1983). «Vie quotidienne, pratiques feminines et histoire». Revue Suisse de Sociologie, 1: 99-109.

Bimbi, F. (1999). Measurament, Quality and Social Changes in Reproduction Time: the Twofold Presence of Woman and Gift Economy. En: Hufton, O.; KRAVARITOU, Y. Gender and the use of time. La Haya: Kluwer Law Int.

Boderías, C.; Carrasco, C.; Alemany, C. (eds.) (1994). Las mujeres y el trabajo. Barcelona: FUHEM-Icaria.

BorGHI, V.; Rosa, M. la (eds.) (1996). «Tempo di lavoro, tempo di vita» (monográfico). Sociologia del lavoro, 58.

Boulin, J.Y.; Lallement, M.; Lefebvre, G.; Silvera, R. (1998). "Temps de travail et modes de vie. Quelques resultats d'une enquête empirique». Futuribles, 237.

BRULleT, C. (2000). «El debat sobre la conciliació ocupació-família dins el marc de la Unió Europea». Revista Catalana de Sociología, 12.

CARDús, S. (2002). Propostes d'intervenció per a la conciliació d'horaris familiars, escolars i laborals. Grup de Recerca ISOR, UAB. Departament de Benestar Social. Generalitat de Catalunya. Informe de investigación.

CARrasco, C. (dir.) (2001). Tiempos, trabajos y género. Barcelona: Publicacions de la Universitat Barcelona.

CarRasco, C.; AlabarT, A.; Mayordomo, M.; Montagut, T. (1998). Las mujeres y el Estado del bienestar: Una aproximación al caso español. Madrid: Instituto de la Mujer.

CARRASQUER, P.; TORNS, T. (2002). «La doble presència femenina i les necessitats de concicliació». Diàlegs, 15.

Carrasquer, P.; Torns, T.; Tejero, E.; Romero, A. (1998). «El trabajo reproductivo". Papers, 55.

Chinchilla, N.; Poelmans, S. (2002). Políticas familiarmente responsables: Informe IFREI 2002. Barcelona: IESE-Universidad de Navarra.

COLECTIVO IOE (1996). Tiempo social contra reloj. Las mujeres y la transformación en los usos del tiempo. Madrid: Instituto de la Mujer. 
COMISSION EUROPÉENNE (1999). Conciliation de la vie professionnelle et familiale et qualité des services de soins: Rapport sur les recherches existant dans l'Union Européenne. Luxemburgo: Commission Européenne.

Duran, M.A. (1986). La jornada interminable. Madrid: Icaria. Editorial Nova Terra.

ELIAS, N. (1985). Time: An Essay. Oxford: Blackwell.

EsCOBEDO, A. (2000). «Les llicències laborals per a mares i pares amb fills menors de tres anys: Una comparació dels sistemes vigents a Dinamarca, Finlàndia i Espanya». Revista Catalana de Sociologia, 12.

GaSPARINI, G. (1998). La dimensione sociale del tempo. Milán: Franco Angeli.

Grossin, W. (1974). Les Temps de la vie quotidienne. París: Albin Michel.

HANTRAIS, L. (2000). Gendered policies in Europe: Reconciling employment and family life. Nueva York: Poligrave.

Heller, A. (1977). La sociología de la vida cotidiana. Barcelona: Península.

Hirata, H.; Senotier, D. (eds.) (1996). Femmes et partage du travail. París: Syros. HÖring, K.H.; Gerahard, A.; Michailow, M. (1995). Time pionners: Flexible Working Time and New Lifestyles. Cambridge (RU): Polity Press.

HufTON, O. (1997). «La investigación europea sobre tiempo y género». Revista Internacional de Sociología, 18.

Hufton, O.; Kravaritou, Y. (1999). Gender and the use of time. La Haya: Kluwer Law Int.

Javeau, C. (1980). «Sur el concep de vie quotidienne et sa Sociologie». Cahier Internationaux de Sociolgie. París: PUF, v. LXVIII: 31-45.

- (1983). «La Sociologie du quotidien: paradigmes et enjeux». Revue Suisse de Sociologie, 1: 21-36.

- (1984). «Prolegómenos metodológicos a una sociología de lo cotidiano». En: RuIZ OlabuÉnAGa, J.I. Estilos de vida en investigación social. Bilbao: Mensajero.

JUNTER-LOISEAU, A.; TOBLER, C. (1999). Reconciliation of domestic and care work with paid work: Approaches in international legislation and policy instruments and in the scientific discours. En: HufTON, O.; KRAVARITOU, Y. Gender and the use of time. La Haya: Kluwer Law Int.

LECCARDI, C. (1994). «Recomponere il tempo: le donne, il tempo, il lavoro». Sociología del Lavoro, 56.

LEFEBVRE, H. (1968, 1975). La vie quotidienne dans le monde moderne. París: Gallimard.

- (1981). Critique de la vie quotidienne III : De la modernité au modernisme. (Pour une métaphisique du quotidien.) París: L'Arche.

LEWIS, J. (ed.) (1998). Gender, social care and welfare state restructuring in Europe. Aldershot: Ashgate.

LÓPEZ, P. (1996). «La construcción de tipologías: metodología de análisis». Papers, 48: 9-29.

LOZARES, C.; LÓPEZ P.; BorRÁS, V. (1998). «La complementariedad del log-lineal y del análisis de correspondencias en la elaboración y análisis de tipologías». Papers, 55: 79-93.

LOZARES, C.; LÓpez, P.; DOMÍNGUEZ, M. (1998). «La articulación de ámbitos sociales a partir de la base temporal». Papers, 55: 115-130.

LOZARES, C.; LOPEZ, P.; MARTÍ, J. (2004). «La relación entre los tiempos y las actividades del trabajo productivo y las actividades del trabajo productivo y reproductivo». Trabajo: Revista de la Asociación Estatal de Centros Universitarios de RRLL y CCTT, 13: 165-186. 
LOZARES, C.; VerD, J.M. (2004). «Modelos de flexibililidad del trabajo y tiempo productivo». Trabajo: Revista de la Asociación Estatal de Centros Universitarios de RRLL y CCTT, 13: 97-113.

MAFFESOLI, M. (1989). «The Sociology of Everyday life. Episthemological Elements». Current Sociology, 37, 1: 1-16.

MendizÁbal, L. (1984). «Sociología de la Vida Cotidiana». En: Ruiz Olabuénaga, J.I. Estilos de vida e investigación social. Bilbao: Mensajero.

Mercure, D.; WallemacQ, A. (ed.) (1988). Les temps sociaux. París: De Boeck.

PraTs, M. (1995). Mujeres, ciudad y tiempo cotidiano: Un enfoque cuantitativo del tiempo de las mujeres de 25 a 50 años en Barcelona. Barcelona: Institut d'Estudis Metropolitans.

Provonost, G. (1996). Sociologie du temps. París: De Boeck.

RemY, J.; Vove, L.; ServaIS, E. (1978). Produire et reproduire? Une sociologie de la vie quotidienne. T. I. Bruxelles: Vie Ouvrière.

RUBERY, J.; FAGAN, C.; SMITH, C. (1994). Changing partners of work and working time in the European Union and the impact of gender divisions. European Comission (DGV-Equal Opportunities Unit).

Salvador JuAn (1995). Les formes élémentaires de la Vie Quotidienne. París: PUF. Samuel, N. (1984). Le temps libre: un temps social. París: Librairie des Méridiens. TABbOni, S. (1986). La rappresentazione sociale del tempo. Milán: Franco Agneli.

TORNS, T. (1994). Women and the distribution of time: A wider vision. Bruselas: IRISEC.

- (2001). «El tiempo de trabajo de las mujeres: entre la invisibilidad y la necesidad». En: CARRASCO, C. (dir.). Tiempos, trabajos y género. Barcelona: Publicacions de la Universitat Barcelona.

- (2004). «Las políticas del tiempo: un reto para las políticas del bienestar». Trabajo, 13: 145-164.

Torns, T.; Carrasquer, P. (1999). «El perquè de la reproducció». Papers, 59.

TORNS, T.; CARRASQUER, P.; BORRÁs, V.; ROCA, C. (2002). El estudio de la doble presencia: una apuesta por la conciliación de la vida laboral y familiar. Bellaterra: QUITDepartamento de Sociología-Instituto de la Mujer. Informe de investigación. VAliente, C. (2000). «Reconciliation policies in Spain». En: Hantrais, L. Gendered policies in Europe: Reconciling employment and family life. Nueva York: Poligrave.

WODZ, J. (1987). Problèmes de la sociologie qualitative. Katowice: Université de Silésie. ZerubaVEL, E. (1981). Hiddens Rhythms: Schedules and Calenders in Social Life. Chicago: University of Chicago Press.

- (1987). "The Language of Time: Toward a Semiotics of Temporality». The Sociological Quartely, 28: 343-356. 